\title{
Colaboración Académica Internacional en Tecnologías de la Información y Docencia Virtual
}

\author{
José O. Valderrama ${ }^{1}$, Ángel Sánchez ${ }^{2}$ y Santiago Urrejola ${ }^{3}$ \\ (1) Dpto. de Ingeniería Mecánica, Universidad de La Serena, La Serena, Chile \\ (2) Dpto. de Ingeniería Química, Universidad de Vigo, 36310 Vigo-España \\ (3) Dpto. De Ciencia de Materiales, Universidad de Vigo, 36310 Vigo-España \\ Universidad de Vigo, Vigo-España
}

\begin{abstract}
Resumen
Se describe y analiza la gestación y puesta en marcha de un proyecto de colaboración académica internacional en tecnologías de la información y docencia virtual entre la Universidad de La Serena en Chile y la Universidad de Vigo en España. El proyecto es financiado por la Agencia Española de Cooperación Internacional para el Desarrollo (AECID) dentro del Programa de Cooperación Interuniversitaria e Investigación Científica entre España e Ibero América. El proyecto, dirigido a la enseñanza de la ingeniería, se enmarca en un convenio marco de colaboración entre ambas universidades, que ha estado vigente por algunos años. Se discute las acciones realizadas a la fecha, pero más importante se analizan con una perspectiva de futuro definiendo las etapas que siguen en este proyecto y resaltando la importancia de estas acciones para la formación de los ingenieros del siglo XXI.
\end{abstract}

Palabras clave: colaboración académica, tecnologías de la información, docencia virtual, AECID

\section{International Academic Collaboration Program on Information Technology and Virtual Teaching}

\begin{abstract}
The founding and development of an international academic collaboration program on information technology and virtual teaching between the University of La Serena in Chile and the University of Vigo in Spain is described and analyzed. The project is financed by the International Development Cooperation Spanish Agency (AECID) within the Interuniversity Cooperation Program between Spain and Ibero America. The project, aimed to improve engineering teaching, is being developed under a general collaboration agreement signed by both universities and that has been active for some years. The tasks done until now are discussed, but most important is that such tasks are analyzed with a long term perspective. The steps that will follow in this project are also defined and the importance of these actions in the formation of the engineers of the XXI century is highlighted.
\end{abstract}

Keywords: academic collaboration, technology information, virtual teaching, AECID 


\section{INTRODUCCIÓN}

La Universidad de La Serena en Chile y la Universidad de Vigo en España, como muchas otras Universidades del mundo realizan frecuentes programas y acciones para proveer ingenieros que contribuyan al desarrollo de sus países y en particular a las regiones donde están insertas, por medio de programas integrales (Userena 2009; Uvigo, 2009). Una educación integral que incluya componentes de las ciencias y de las humanidades en una forma balanceada, que permitan formar ingenieros más creativos, proactivos, con buenas bases en comunicación y con una componente ética sólida para que en su futuro trabajo profesional puedan comprender la importancia e impacto social y económico de su labor, es de especial importancia en el competitivo mundo en que vivimos (Otero-Gephardt et al., 2005). En el mundo globalizado de hoy la comunidad a la que sirve un ingeniero es mucho mas grande que en el pasado y hay pocos trabajos de ingeniería que no tengan hoy en día un impacto global. Sin embargo, a pesar de los espectaculares cambos tecnológicos de los últimos años, la educación en ingeniería no ha experimentado cambios notables, según algunos autores (Lang et al., 1999). Los cambios han sido mas notorios en algunos países iberoamericanos en los que algunas universidades han adoptado diversas acciones para cambiar la forma en que sus docentes enseñan y sus estudiantes son formados. La relación entre universidades latinoamericanas y otras de los países desarrollados y el intercambio académico y estudiantil como parte de la formación profesional ha sido una acción de especial importancia en este desarrollo y algunas universidades lo han adoptado como una política institucional que fomentan y apoyan.

El concepto de cooperación internacional en el ámbito académico no es nuevo. Lo que si parece más novedoso es la identificación e implementación de oportunidades internacionales que ofrecen universidades líderes en el mundo y los propios gobiernos. La economía global ha determinado una nueva forma de pensar y en esto los ingenieros han sido los primeros llamados a adaptarse al nuevo escenario. Por lo tanto, modificar y adaptar los programas de estudio es una tarea y obligación permanente de las universidades para que los ingenieros del futuro cercano tengan una visión amplia para entender el complejo mundo en que estarán insertos (Wormley, 2004, Briggs, 2007; Wolf, 2007). La globalización de la educación en ingenieria es esencial para forma ingenieros para un mundo también económicamente globalizado. El portal de la UNESCO sobre Enseñanza superior y en particular sobre Ingenieria, Tecnología y Medio ambiente (UNESCO, 2009) es probablemente el mejor enlace para la transferencia de información, en la enseñanza de la ingenieria. La globalización de la ingenieria puede hacer que temas candentes con complejas aristas políticas, sociales y económicas, puedan ser abordados con eficiencia y rapidez. Es en este contexto de adaptación de nuevas tecnologías que se desarrolla el proyecto y en el cual se han realizado ya acciones concretas, como se describe más adelante.

Entre los cambios más importantes de los últimos años y a los que las universidades han debido adaptarse, y deben hacerlo pronto si aún no o han hecho, es al uso de tecnologías actuales de información y comunicación. Un estudiante que usa esta tecnologías en su proceso de aprendizaje está haciendo uso de lo que en forma genérica se conoce como aprendizaje electrónico, más conocido por su nombre en Inglés, e-learning. Existen numerosas formas de transmisión de información y de enseñanza-aprendizaje que caen en esta categoría de e-learning: simple acceso a Internet, búsqueda a través de bases de datos, programas tutoriales en línea, control a distancia de equipos de laboratorio o de software, herramientas de simulación y diseño, análisis de datos en programas en línea, experimentos de laboratorio, foros y blogs, entre tantos otros. La cantidad de formas y métodos posibles de crear usando las tecnologías modernas es inimaginable, en especial porque cuando ya una forma de interacción ha madurado, se ha generalizado y adoptado, aparece una nueva aplicación tecnológica que supera todo lo realizado y permite ir más allá en el método desarrollado. Una buena fuente de información relacionada con e-learning es el libro Changing Higher Education (Ashwin, 2006), y en particular el capitulo 6 Elearning in Higher Education (Laurillard, 2006). Laurillard resume los avances en las nuevas tecnologías en las últimas décadas las compara con las tecnologías o medios que reemplaza y clasifica los avances por la función que apoyan, simplifican o generan. La Tabla 1, adaptada de Laurillard, resume estos conceptos. 
La docencia virtual tiene una importante aplicación en las Universidades de todo el mundo. Hoy en día, no es aceptable la inclusión de un curso en un campus virtual (plataforma de e-learning), sin una virtualización de la enseñanza de los documentos, de modo que los alumnos pueden acceder al curso regular y complementar la enseñanza en clases de la universidad (Casar et al., 2007). El e-learning ha experimentado un profundo cambio durante los últimos diez años. Inicialmente, el aprendizaje electrónico era "algo" nuevo, y la carga a la plataforma de las presentaciones en PowerPoint utilizadas por el profesor en clase se consideró suficiente. Pero la evolución del elearning ha sido muy rápida y hoy en día la demanda de cursos virtuales de alta calidad, con un fácil acceso, y con el uso de la mayoría de las funcionalidades de la plataforma para manejar información ha crecido en forma muy rápida (Wolf, 2007; South y Monson, 2009). La virtualización de la enseñanza a través de una plataforma e-learning resuelve en parte los problemas generados por la distancia entre ambas universidades, como es el caso del proyecto que se discute en este trabajo. La experiencia de la Universidad de Vigo es amplia en este campo siendo la primera Universidad de habla castellana seleccionada por ItunesU para virtualizar sus contenidos y ponerlos a disposición de los potenciales usuarios en Internet (Itunes, 2009). La experiencia es más nueva para la Universidad de La Serena, por lo que este proyecto es de especial significado para avanzar en el uso de nuevas tecnologías para la formación de los futuros ingenieros titulados en la Universidad de La Serena.

Tabla 1: Nuevas tecnologías de la información, comunicación y transmisión de conocimiento como apoyo al aprendizaje (adaptado de Laurillard, 2006)

\begin{tabular}{|c|c|c|c|}
\hline Periodo & Nueva tecnología & $\begin{array}{l}\text { Tecnología antigua } \\
\text { equivalente }\end{array}$ & $\begin{array}{l}\text { Función que apoyan, } \\
\text { simplifican o generan }\end{array}$ \\
\hline \multirow[t]{2}{*}{$\begin{array}{l}\text { Los años } \\
70\end{array}$} & $\begin{array}{l}\text { Computadores } \\
\text { interactivos }\end{array}$ & Escritura & $\begin{array}{l}\text { Medio para articular y transmitir } \\
\text { ideas }\end{array}$ \\
\hline & $\begin{array}{l}\text { Discos duros locales } \\
\text { y disquetes }\end{array}$ & Papel & Almacenamiento de información \\
\hline \multirow[t]{3}{*}{$\begin{array}{l}\text { Los años } \\
80\end{array}$} & $\begin{array}{l}\text { Interfaces WIMP } \\
\text { (Windows, Iconos, } \\
\text { Menús, Punteros) }\end{array}$ & $\begin{array}{l}\text { Contenidos, índices, } \\
\text { numero de paginas }\end{array}$ & Facilitar acceso a contenidos \\
\hline & Internet & Impresos & $\begin{array}{l}\text { Distribución masiva de } \\
\text { contenidos }\end{array}$ \\
\hline & Multimedia & $\begin{array}{l}\text { Fotografía, sonido y } \\
\text { películas }\end{array}$ & $\begin{array}{l}\text { Formas elaboradas de } \\
\text { presentación }\end{array}$ \\
\hline \multirow[t]{5}{*}{$\begin{array}{l}\text { Los años } \\
90\end{array}$} & $\begin{array}{l}\text { Red Mundial } \\
\text { (Worldwide Web) }\end{array}$ & Bibliotecas & Amplio acceso a contenidos \\
\hline & $\begin{array}{l}\text { Computadores } \\
\text { personales (laptops) }\end{array}$ & Libros en papel & $\begin{array}{l}\text { Acceso personal, portátil a } \\
\text { contenidos }\end{array}$ \\
\hline & $\begin{array}{l}\text { Correo electrónico } \\
\text { (email) }\end{array}$ & $\begin{array}{l}\text { Servicios postales de } \\
\text { correos }\end{array}$ & $\begin{array}{l}\text { Comunicación escrita a } \\
\text { distancia }\end{array}$ \\
\hline & $\begin{array}{l}\text { Máquinas de } \\
\text { búsqueda }\end{array}$ & Servicios bibliográficos & $\begin{array}{l}\text { Acceso eficiente a contenidos } \\
\text { extensos }\end{array}$ \\
\hline & Banda ancha & $\begin{array}{l}\text { Teléfonos difusión } \\
\text { (broadcasting) }\end{array}$ & Velocidad de la comunicación \\
\hline \multirow[t]{2}{*}{$\begin{array}{l}\text { Desde el } \\
2000\end{array}$} & $\begin{array}{l}\text { Móviles de } 3^{a} \\
\text { generación ( } 3 G \\
\text { Móviles) }\end{array}$ & Paperbacks & $\begin{array}{l}\text { Acceso masivo y económico a } \\
\text { contenidos }\end{array}$ \\
\hline & Bitácoras (Blogs) & Panfletos & Publicación personal masiva \\
\hline
\end{tabular}




\section{EL CONVENIO MARCO}

El Convenio Marco firmado por las Universidades de Vigo-España y La Serena-Chile es un documento general y constituye la base de todos los programas y proyectos específicos entre grupos de distintos departamentos de cada Universidad. El Convenio define el formato por el que cada proyecto específico se regirá, incluyendo: objetivos, descripción de las actividades a realizar, formulación del proyecto y obtención de financiamiento adicional, procedimientos de publicación de documentos producidos en virtud de un determinado proyecto, y asuntos de propiedad intelectual si los hubiere. Todos los acuerdos específicos no pueden involucrar contratos de personal o relaciones laborales. Si hubiera una situación de ese tenor es materia da cada universidad y no se puede invocar el convenio para esos fines.

El convenio marco fue firmado por los rectores de ambas universidades a comienzos del año 1997 y se mantiene activo. El acuerdo establece las obligaciones, los derechos y las limitaciones de las partes para la ejecución de las actividades de colaboración establecidas. La introducción del convenio establece lo siguiente: "Ambas partes se reconocen entre sí capacidad jurídica suficiente y poder bastante para obligarse en este Convenio y, a tal efecto, exponen: que ambas entidades consideran de interés común promover la cooperación y la cooperación y la colaboración para el desarrollo científico y tecnológico de Galicia en España y de la Región de Coquimbo en Chile, procurando una aplicación practica del conocimiento en provecho de nuestra sociedad; que es deseo de ambas Instituciones la firma de un Acuerdo Marco de Colaboración que instrumente y regule sus relaciones mediante la eventual suscripción de Convenios Específicos. Por esto, ambas partes acuerdan suscribir el presente Acuerdo Marco de colaboración, conforme a las siguientes cláusulas..."

El resto del convenio establece otros aspectos relacionados con el tipo de actividades a desarrollar, con publicaciones conjuntas, con eventuales patentes, asuntos económicos y con resolución de conflictos. El convenio marco sin duda que facilita la postulacion a proyectos y dineros externos que usualmente requieren algún tipo de vínculo previo entre las universidades que buscan apoyo. Hasta ahora el convenio se ha concretado en intercambio de académicos, cursos cortos dictados en España, proyectos específicos entre colegas del Dpto. de Ingeniería Química de la Universidad de Vigo y del Departamento de Ingeniería Mecánica de la Universidad de La Serena. Durante estos años el convenio marco ha servido para facilitar la burocracia y conseguir apoyos externos concretando varias actividades de intercambio académico. Colegas de la Universidad de Vigo han visitado la Universidad de La Serena y colegas de la Universidad de La Serena han visitado la Universidad de Vigo. En ambos casos se han desarrollado diversas actividades, tales como presentación de cursos de posgrados, elaboración de proyectos, asistencia a congresos internacionales y publicaciones conjuntas (Valderrama et al., 1997, 1998, 1998a, 1999).

La Universidad de La Serena está aún en una etapa crítica de su desarrollo, con varias carreras en proceso de acreditación y algunas ya acreditadas. Hay por lo tanto varios programas buscando formas novedosas y eficientes de mejorar la docencia y la investigación como requisitos básicos para la necesaria acreditación. Ha habido algunos avances en el uso de tecnologías de la información pero han sido de poca envergadura. En investigación, los avances no son suficientes y se traducen en un índice muy bajo de proyectos y de publicaciones. En ambos casos los recursos son pocos y los incentivos no llegan a quienes pueden contribuir a mejorar los índices de productividad docente y de investigación, aunque hay algunos esfuerzos (DIULS, 2009). El proyecto que se analiza en lo que sigue se enmarca en este convenio marco y, aparte de mantener activo el convenio, aportará en mejorar nuestros métodos de enseñanza y facilitar el aprendizaje de los alumnos usando tecnologías modernas.

\section{EL PROYECTO ACTUAL}

La docencia compartida entre equipos de Universidades de distintos continentes suma a los problemas normales de cualquier tipo de docencia el hecho de estar ubicados en distintas áreas geográficas separadas por grandes distancias. En septiembre del año 2008 un grupo de 
investigación de la Universidad de Vigo y uno de la Universidad de la Serena se planteó la posibilidad de crear un posgrado en simulación en ingeniería de procesos que compartiera las experiencias de ambos grupos en investigación y docencia. Los objetivos de este proyecto fueron conseguir una colaboración estable en el ámbito de la simulación de procesos entre la Universidad de La Serena y la Universidad de Vigo. Ello permitiría el fortalecimiento de las relaciones entre ambas instituciones que se plasmaría en acciones concretas de colaboración en docencia tanto en grado como en posgrado.

Uno de los problemas fundamentales en la colaboración actual es el elevado coste tanto económico como en tiempo que se debe asumir por parte de los docentes españoles para impartir cursos en La Serena y los docentes chilenos para impartir cursos en Vigo. Es así como se obtiene apoyo de la Agencia Española de Cooperación Internacional para el Desarrollo (AECID) dentro del Programa de Cooperación Interuniversitaria e Investigación Científica entre España e Ibero América, proyecto B/021969/08. La idea básica y primaria del proyecto fue el Desarrollo de Cursos Posgrado Virtuales sobre Simulación en Ingeniería de Procesos aprovechando las ventajas comparativas y potencialidades de cada grupo.

\section{Antecedentes de los grupos}

El grupo de investigación EQEA (Ingeniería Química, Energética e Ambiental de la Universidad de Vigo) ubicado en la Escuela Técnica Superior de Ingenieros Industriales, es un grupo multidisciplinar formado por investigadores de tres áreas de conocimiento: i) Ingeniería Química, ii) Máquinas y Motores Térmicos, y iii) Ingeniería de Materiales. El grupo tiene una dilatada experiencia en actividades de I+D y desde el año 2000 trabaja con programas de simulación para dar soluciones a empresas del sector en el diseño de plantas químicas (proyecto Regadi), a partir del año 2004 en el que el grupo de investigación crea un subgrupo dedicado al estudio del hidrógeno y las pilas de combustible, que emplean varios software de simulación comerciales para el desarrollo de proyectos de I+D+I con distintas empresas gallegas y con el apoyo de la Xunta de Galicia.

Por su parte el equipo de la Universidad de La Serena posee amplia experiencia en el campo de la simulación de procesos industriales, métodos numéricos aplicados al modelado y simulación de procesos termodinámicos y energéticos, y en docencia de pregrado y de posgrado. Desde hace varios años el grupo se ha especializado en procesos de separación y simulación de procesos industriales. En estos temas el grupo ha publicado extensamente en la literatura técnica internacional y ha dictado cursos en varios países de Ibero América. Destacables entre estos son cursos industriales en Argentina, Perú, Costa Rica y Venezuela, dirigidos a profesionales involucrados en procesos reales de planta que requieren herramientas de simulación de procesos. Igualmente el grupo enfatiza su trabajo en técnicas numéricas y computacionales necesarias para la simulación de procesos de separación y estimación de propiedades de fluidos para la simulación de procesos.

\section{Los Contactos y Visitas}

Tras las primeras reuniones que los componentes de ambos grupos de investigación mantuvieron durante la visita que los profesores de la Universidad de Vigo realizaron a la Universidad de la Serena, se llegó a la conclusión de que uno de los principales escollos de un posgrado a ambos lados del Atlántico era evidentemente la distancia. Por ello, con el fin de reducir los problemas que la distancia produce se propone en el proyecto el desarrollo de un modelo de docencia virtual. En estas conferencias se presentó la infraestructura de producción de contenidos docentes y videoconferencia así como el proceso de implantación realizado en la Universidad de Vigo usando las nuevas tecnologías. Esto permitiría ampliar los horizontes de este proyecto no sólo a la enseñanza de las disciplinas de la ingeniería de procesos sino también al resto de las de las ingenierías de la Universidad de La Serena. Fue así como se programó la visita por parte de dos profesores equipo de la Universidad de Vigo al que se sumaron dos empresarios vigueses cuyas empresas dan apoyo a la docencia virtual en la Universidad de Vigo. En esta visita, se expusieron las posibilidades de docencia virtual y se planificó trabajo futuro. Entre las actividades planificadas 
fue la visita de un investigador chileno a Vigo para realizar en forma concreta actividades de grabación de documentos docentes, como se expone en la sección siguiente. Se acordó que lo más inmediato y factible de realizar era la realización de una actividad concreta de especial interés para ambas partes y que se podía concretar durante la visita: las píldoras docentes. Esta actividad es descrita en la próxima sección de este trabajo.

Dadas las dificultades surgidas a la hora de planificar los desplazamientos entre Chile y España para la realización de contenidos docentes los coordinadores del mismo, animados por los buenos resultados obtenidos, y las implicaciones que en un futuro puede tener un proyecto de cooperación educativa, han decidido solicitar una prórroga de dicho proyecto a la que han sumado nuevos investigadores para enriquecer los contenidos del mismo y seguir avanzado en esta línea que consideran como el futuro de la docencia.

\section{Avances en el Grupo Español}

El año 2003 la Universidad de Vigo se inició un plan de introducción de nuevas tecnologías educativas en el marco del proyecto europeo TORGA.net (Trans Red Gallega de Portugal) [Torga, 2009], proyecto que permitió crear una red de comunicaciones avanzadas de última generación que interconecta los campus de Vigo, Ourense, Pontevedra, y Santiago de Compostela en España y Braga y Guimaraes en Portugal, mediante línea de fibra óptica de alta capacidad. Como resultado de este y otros proyectos el uso de las infraestructuras informáticas disponibles ha ido cambiando las formas de enseñanza-aprendizaje. Una de las más exitosas herramientas han sido las video conferencias, que debido a su creciente utilización surgió la necesidad de registrar ese material de aprendizaje de un modo compatible con el español ARCA (Goyanes, 2008; ARCA, 2009). En la actualidad la capacidad de producción de la Universidad de Vigo es de 600 horas al año de multimedia listo para usar con objetos tales como vídeo, audio, conferencias, screencasts y presentaciones. Entre estos objetos la grabación vídeo, que parece como solución viable en cursos virtuales, presenta la menos dos problemas principales: i) problemas inherentes a la grabación de personas en un aula y la sincronización con los demás elementos utilizados en la clase, tales como audio, proyecciones, y tablero, entre otros; y ii) problemas con el tamaño de los archivos generados. Una clase de una hora (o incluso más) es un archivo muy pesado, y por lo general, los estudiantes no visualizan el video completo. Estos problemas derivaron en vídeos de calidad baja y poco interés por los usuarios, los estudiantes, cuestión que produjo los necesarios cambios para mejorar los productos multimedia generados.

Hoy en día se dispone medios técnicos y humanos que permiten la grabación y edición de contenidos multimedia, producción de un archivo de vídeo de alta calidad. En la Universidad de Vigo, este archivo se codifica en Adobe Flash Media Server con tres velocidades de transmisión de datos (bit rate) para que el sistema se pueda adaptar automáticamente la velocidad de bits de contenido para el ancho de banda disponible por el usuario. La fase de producción llega a su fin con un archivo maestro, que normalmente es en H264, DV o HDV que se almacena para uso posterior, un archivo de vídeo de Windows Media que alimenta el sistema de distribución y una tabla de metadatos que alimenta el sistema de publicación. El uso de estos sistemas de grabación en un entorno como un campus dotado de una moderna red que nos permite llevar a cabo, a muy bajo costo, las tareas, que hasta ahora sólo eran viables para las estaciones de televisión comercial. Junto con estas mejoras se optó por objetos más atractivos para los usuarios, de más corta duración y mas focalizados en aprendizajes de conceptos complejos, las píldoras docentes, que se describe en lo que sigue.

\section{PÍLDORAS DOCENTES}

De acuerdo con la definición de la RLO-CETL, una píldora de aprendizaje es un objeto reutilizable de aprendizaje, es decir, una entidad digitalizada, que puede ser utilizada, reutilizada o referenciada en la tecnología, con el apoyo de aprendizaje (Wiley, 2009; South y Monson, 2009). Entre las ventajas de las píldoras docentes es el poco tiempo que lleva su grabación y en la mayoría de los casos el hecho de poder volver a utilizar presentaciones de PowerPoint o cualquier otra herramienta de aprendizaje en forma de table PC o una pizarra electrónica, que el área de la pantalla de la píldora de aprendizaje puede contener. Otra ventaja es la presencia de la imagen de 
los docentes en la píldora no genera problemas relacionados con los derechos de autor de contenidos compartidos entre docentes de la misma materia. El esquema de una píldora docente se muestra en las fotografías. Según el Polimedia el formato aún no está normalizado, el que usa la Universidade de Vigo se llama como 1024P + P. Este formato nos permite combinar una imagen tomada del PC (presentación de powerpoint, pizarra electrónica...) y la imagen de vídeo del profesor o ponente en el mismo video. El producto final es una imagen en movimiento con una resolución de 1024 x 768 que se compone del profesor, su voz y el programa utilizado incluidos los movimientos del puntero del ratón (Fig. 1. Esto sistema es muy valioso en las explicaciones científicas en las que el profesor está señalando una zona determinada en un gráfico con el ratón o una formación fisiológica en particular.

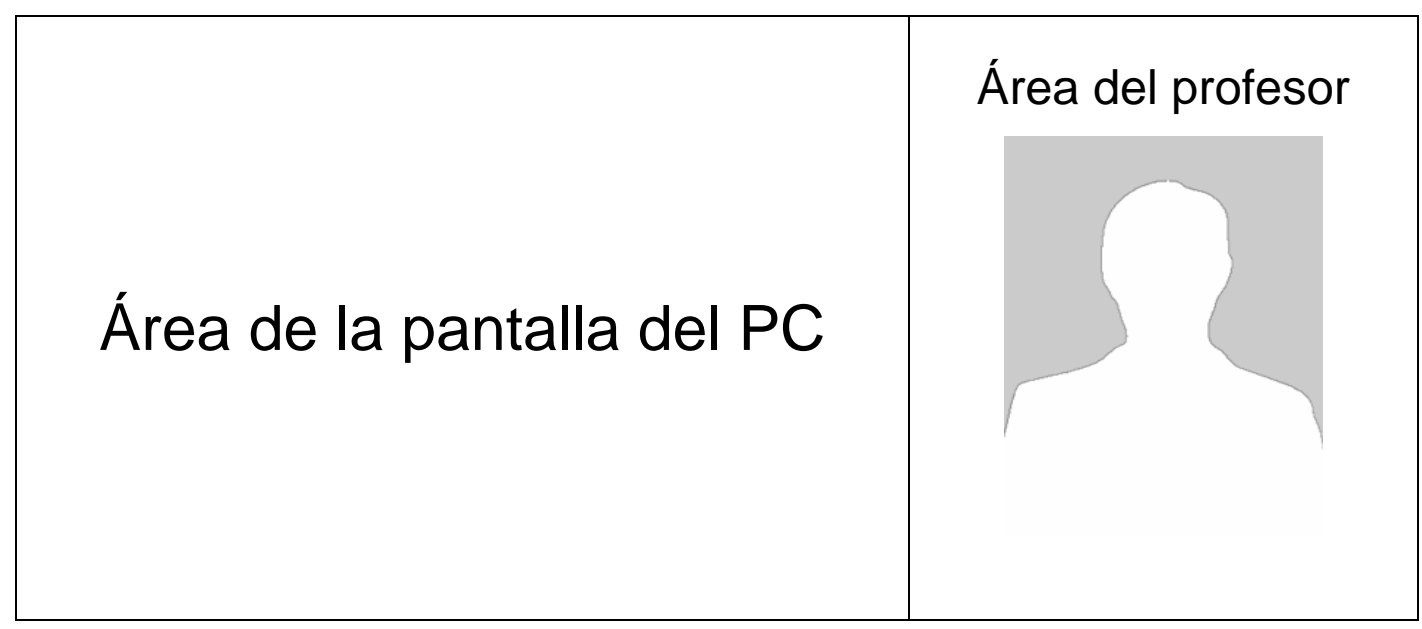

Fig. 1: Esquema de la píldora docente mostrando las áreas de pantalla y del profesor

Tradicionalmente, el contenido de una píldora viene en un trozo de video de entre 10 y 15 minutos y son objetos autónomos, pudiendo ser tomados de forma independiente y reutilizable. De esta manera un mismo objeto, puede ser utilizado en múltiples contextos para múltiples propósitos, en diferentes cursos y a las que se puede recurrir en cualquier momento desde cualquier parte donde el usuario se encuentre. Este tipo de objetos de aprendizaje, al que pertenecen las píldoras docentes, se pueden agrupar en colecciones más grandes de contenido, incluyendo estructuras de los cursos tradicionales, etiquetados con metadatos lo que permite una búsqueda más expedita y rápida. La Tabla 2 muestra resume las características las características básicas y algunas ventajas de las píldoras docentes.

Tabla 2: Algunas características básicas y algunas ventajas de las píldoras docentes

\begin{tabular}{|l|l|}
\hline Características & Ventajas \\
\hline Objetos autónomos & Poco tiempo que lleva su grabación \\
Enfocados a un tema & Puede utilizar presentaciones ya disponibles \\
Indivisibles & Puede ser utilizado en múltiples contextos \\
Dirigidos a una audiencia especifica & Puede ser usado para múltiples propósitos \\
Agrupables & Puede ser usado en diferentes cursos \\
\hline
\end{tabular}

\section{Píldoras realizadas}

En el mes de Junio de 2009 el autor chileno visitó la Universidad de Vigo y tuvo la oportunidad de grabar algunas píldoras docentes que estarán disponibles en la Web del proyecto (actualmente en construcción), junto con píldoras grabadas por el equipo de la Universidad de Vigo. La Figura 1 muestra ejemplos de estas píldoras, y que es la forma en que los alumnos usuarios las ven. Las píldoras grabadas hasta la fecha tienen una duración media de 12 minutos y todas usan presentaciones en power point como texto de apoyo para las explicaciones de los conceptos que 
trata la píldora. Dado el carácter experimental de este proyecto docente se decidió con el fin de ver su respuesta entre el alumnado de ambas instituciones editar unas píldoras de carácter mas general y otras de temas básicos de ingeniería química estas píldoras estarán próximamente disponibles en la Web del proyecto (http://www.santiagourrejola.com/B02196908). La Tabla 2 muestra un listado de píldoras actualmente disponibles y otras en proceso para ser grabadas durante el año 2010.

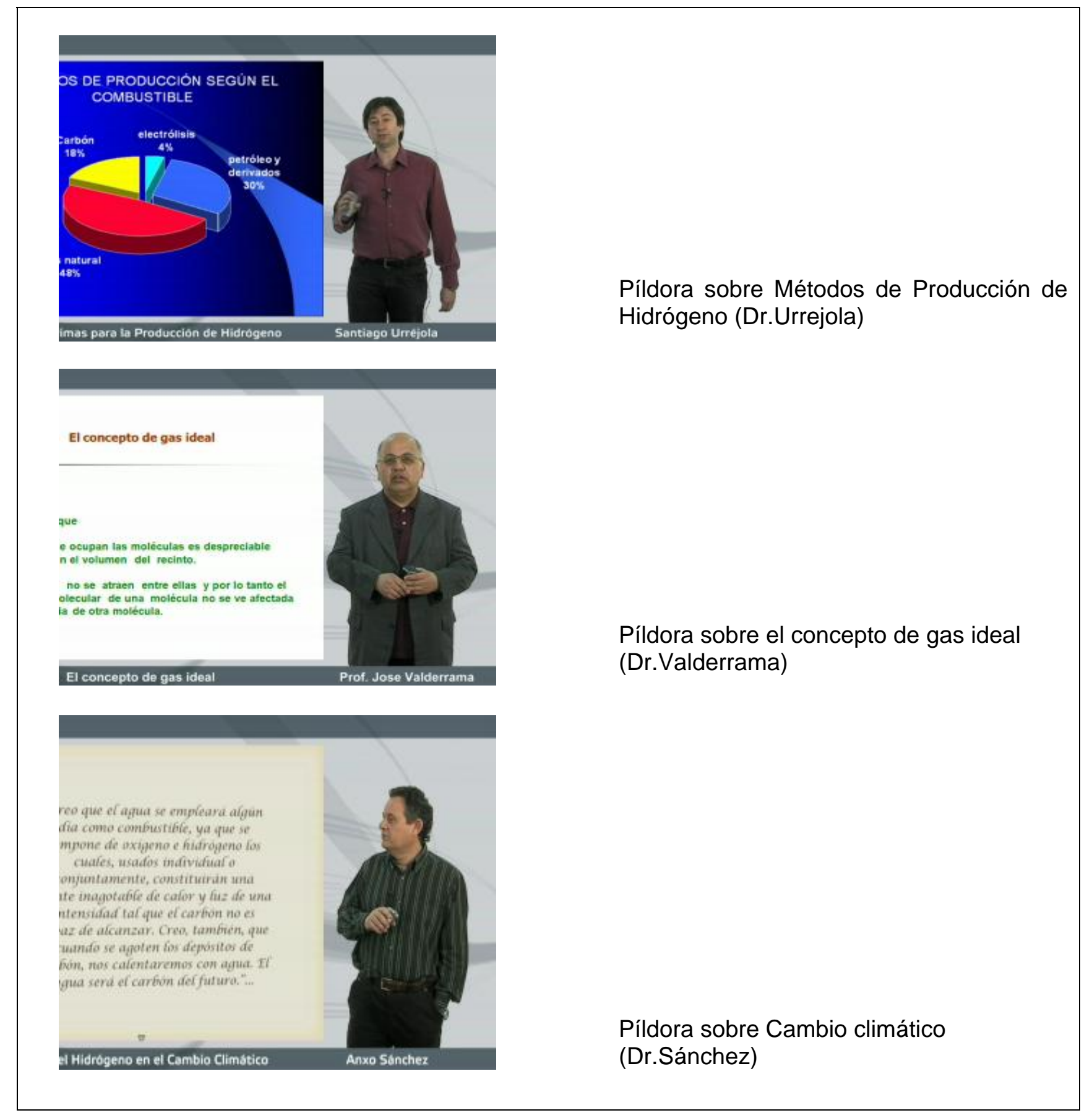

Figura 1: Ejemplos de píldoras docentes realizadas en la Universidad de Vigo por miembros del equipo de intercambio de ambas universidades

\section{Las etapas que siguen}

Los grupos planifican nuevas visitas de los integrantes del mismo a las Universidades para seguir avanzando en la consolidación de este tipo de herramientas docentes y la grabación de nuevos píldoras que permitan comprobar experimentalmente con los alumnos de ambas universidades la 
validez que este tipo de metodología tiene para la enseñanza futura de cursos entre Universidades separadas por grandes distancias.

Durante el año 2010 se dictará en Vigo un curso sobre Propiedades De Fluidos Para La Simulación De Procesos y en La Serena se dictará un curso sobre Energías Renovables. Se planifica la realización de varias píldoras docentes (como se muestra en la Tabla 2), y de conferencias sobre nuevas tecnologías. Sin embargo, más importante que estas realizaciones es el contexto en que ellas se realizan. Esto es que los importantes avances realizados hasta la fecha en la universidad de Vigo en materias de uso de tecnologías modernas en la enseñanzaaprendizaje, es su transferencia a una Universidad chilena, donde existen las condiciones para su realización. El grupo de La Serena por su parte aportara con su experiencia en la simulación de procesos industriales al que se incorporaran modernas tecnologías de aprendizaje.

Tabla 2: Algunos temas de píldoras

\begin{tabular}{|c|l|l|l|}
\hline $\mathbf{N}^{\mathbf{0}}$ & Píldoras ya realizadas & $\mathbf{N}^{\mathbf{0}}$ & Píldoras a realizar el año 2010 \\
\hline 1 & Equilibrio químico, Introducción & 13 & La Primera Ley de la Termodinámica \\
\hline 2 & Equilibrio Acedo-Base & 14 & La segunda Ley de la Termodinámica \\
\hline 3 & El concepto de mol y masa molecular & 15 & Temperatura de Bulbo Húmedo \\
\hline 4 & El concepto de Gas Ideal & 16 & El Diagrama Sicrométrico \\
\hline 5 & El concepto de energía & 17 & La Temperatura Absoluta \\
\hline 6 & $\begin{array}{l}\text { El Papel do Hidrogeno no Cambio } \\
\text { Climático }\end{array}$ & 18 & $\begin{array}{l}\text { El Concepto de Energía Interna y } \\
\text { Entalpía }\end{array}$ \\
\hline 7 & Las Pilas de Combustible & 19 & Presión de Vapor y Transición de Fases \\
\hline 8 & Métodos de Producción de Hidrógeno & 20 & Capacidad Calorífica \\
\hline 9 & $\begin{array}{l}\text { Materias Primas para Producir } \\
\text { Hidrógeno }\end{array}$ & 21 & Ecuaciones de Estado \\
\hline 10 & $\begin{array}{l}\text { Distribución de Hidrógeno e } \\
\text { Infraestructuras }\end{array}$ & 22 & La ecuacion de van der Waals \\
\hline 11 & $\begin{array}{l}\text { Simulación dinámica de un vehículo } \\
\text { eléctrico híbrido con hidrógeno }\end{array}$ & 23 & Reacciones de Combustion \\
\hline 12 & $\begin{array}{l}\text { Modelado e simulacion dunha bomba } \\
\text { de calor xeotérmica }\end{array}$ & 24 & Simulación en Ingeniería Química \\
\hline
\end{tabular}

\section{CONCLUSIONES}

La discusión presentada y los avances a la fecha permiten obtener las siguientes conclusiones generales: i) La colaboración entre Universidades que tanto recorrido tiene en materia de investigación se hace en el nuevo marco mundial de globalización necesario en materia de docencia; ii) Las nuevas herramientas de virtualización de contenidos y teledocencia (e-learning) hace cada vez mas factible la colaboración entre Universidades separadas por grandes distancias; iii) Para conseguir el futuro objetivo de un posgrado virtual inter universitario es necesario experimentar con las muevas herramientas de e-learning; y iv) El proyecto de Cooperación Interuniversitaria B/021969/08 (AECID) pretende consolidar la relación existente entre las Universidades de La Serena y Vigo en materia de formación.

\section{AGRADECIMIENTOS}

Los autores agradecen a sus respectivas universidades por el apoyo prestado para concretar las visitas y al proyecto B/021969/08 financiado por la Agencia Española de Cooperación Internacional para el Desarrollo (AECID) dentro del Programa de Cooperación Interuniversitaria e Investigación Científica entre España e Ibero América por el apoyo económico. 


\section{REFERENCIAS}

ARCA, Project website (2008). ARCA Project, Retrieved May, 2009, from https://apu.uc3m.es/arca/ (2009)

Ashwin, P. (editor) Changing Higher Education: The Development of Learning and Teaching, $1^{\mathrm{a}}$ ed., pag. 79, Routledge Pub., Taylor and Francis, New York, USA (2006)

Casar, A., S. Urrejola, C. Cameselle, A. Sánchez, Edulearn Virtualization of a Thermodynamic Course in a Renewable Energy Master's Degree, E.T.S. de Ingenieros Industriales. Universidad de Vigo, Vigo, España. Polimedia Website, retrieved Mayo, 2009 from: http://polimedia.upv.es (2009)

Briggs, C.L., Curriculum Collaboration: A Key to Continuous Program Renewal, The Journal of Higher Education - Volume 78, Number 6, November/December 2007, pp. 676-711

DIULS, Dirección de Investigación, Universidad de La Serena, http://www.userena.cl/investigacion/, (2009)

Goyanes, V., Arturo Casar, Jose María Pousada, Ruben Gonzalez IPTV for Educational Multimedia Content distribution. Uvigo-TV, ARCA and PuMuKIT projects in Spain. EUNIS 2008, [online] http://eunis.dk/papers/p69.pdf.)

ITUNES, Free Application for Your Mac or PC, http://itunes.uvigo.es

Lang, J.D., Susan Cruse, Francis D. Mcvey and John Mc Masters, Industry Expectations of New Engineers: A Survey to Assist Curriculum Designers, Journal of Engineering Education vol 88(1), 43-51 (1999)

Laurillard, D., E-learning in Higher Education, cap 6 en libro editado por Ashwin (2006)

Otero-Gephardt, Z., J.O. Valderrama and C.V. Ponce, Rowan University and La Universidad de La Serena, An International Collaboration for Teaching and Research, Proceeding $S$ of the 2005 American Society for Engineering Education Annual Conference \& Exposition (2005).

Pildoras, http://tv.uvigo.es/es/pildoras.html (2009)

Polimedia Website. Retrieved May, 2009 from: http://polimedia.upv.es/

TORGA, http://www.cesga.es/content/view/674/86/lang,es

UNESCO, http://portal.unesco.org/education/es/ev.php-URL_ID=6993\&URL_DO=DO_TOPIC\& URL_SECTION =201.html (2009)

Userena, Universidad de La Serena, Chile, www.userena.cl, 2009

Uvigo, Universidad de Vigo, España, www.uvigo.es, 2009

Valderrama, J.O., B. Orge, M. Iglesias y José Tojo, "Aplicación del Simulador Comercial ChemCAD III en la Predicción del Equilibrio Líquido-Vapor en Sistemas Ternarios Complejos", XXVI Reunión Bienal de la Real Sociedad Española de Física, Las Palmas de Gran CanariaEspaña, Septiembre-Octubre (1997).

Valderrama, J.O., M. Iglesias e I. Garcés, "El Software ChemCAD III y su Aplicación al Cálculo de Procesos Complejos de Separación", presentado en el 1er Congreso Panamericano de Informática" en el marco de la XXV Convención de la UPADI, Lima - Perú (Noviembre de 1998).

Valderrama, J.O., Beatriz Orge, Miguel Iglesias y José Tojo, Equilibrio Líquido-Vapor en Sistemas Ternarios Complejos, Inf. Tecnol., 9(1), 107-110 (1998a).

Valderrama, J.O., P.F. Arce y M. Iglesias, "Regla Modificada de Wong y Sandler para Sistemas Líquido+Gas Altamente asimétricos a Alta Presión". Congreso EQUIFASE`99, Vigo - España (1999) 
South, J. B. y Monson, D. W. A university-wide system for creating, capturing, and delivering learning objects. In D. A. Wiley (Ed.), The instructional use of learning objects. Retrieved May, 2009 at: http://reusability.org/read/chapters/south.doc

Wiley, D. A. Connecting learning objects to instructional design theory: a definition, a metaphor, and a taxonomy. In: D.A. Wiley (Ed.), The instructional use of learning objects. Retrieved May, 2009 from:http://reusability.org/read/chapters/wiley.doc .).

Wolf, P., A model for facilitating curriculum development in higher education: A faculty-driven, data-informed, and educational developer-supported approach, New Directions for Teaching and Learning, 112, Pages $15-20$ (2007)

Wormley, D.N., Challenges in Curriculum Renewal, International Journal. Engineering Education, 20(3), 329-332 (2004). 